\title{
Antihyperlipidemic Activities of Isolated bio Compounds of Aegle Marmelos
}

\author{
A.M.Krupanidhi ${ }^{1}$, Kalleshappa C.M. $^{2}$, Ashok R.Chanchi ${ }^{1}$, \\ Prakash Dabadi ${ }^{1}$, Akshara $^{1}$ \\ ${ }^{I}$ Department of Pharmacology,Bapuji Pharmacy College, Davangere -577004, Karnataka, India. \\ ${ }^{2}$ BIET-Engineering College Davangere-577004 Karnataka, India.
}

\begin{abstract}
The present work was done to study qualitative investigation of extractability of the useful compounds from Aeglemarmelos leaves via supercritical fluid extraction technique (SFE) and to evaluate antihyperlipidemic activity of Aeglemarmelos $(\mathrm{Am})$. The obtained extract and fractions were treated on Triton induced rat models and the results showed lowering the lipid profile levelin the experimental models by in-vivo methods. The fractions of Aeglemarmelos $(\mathrm{AmF1})$ also exhibited excellent anti hyperlipedimic property at $3^{\text {rd }}$ week and also lowering the serum cholesterol, triglycerides in Triton induced rats. The most important ingredients present in plant communities turn out to be alkaloids, terpenoids, steroids, phenols glycosides and tannins are responsible for monitoring the normal lipid profile in Triton induced rats due to LA of lecithin acetyl transferase (LCAT), which regulates lipids concentration. The LCAT plays a key role in the incorporation of free cholesterol into HDL (this may increase HDL) and transferring it back to VLDL and LDL which are taken back later in liver cells.The changes in the level of serum lipids in experimental rats areillustrated in (Table 1). The total Cholesterol, TG1, LDL and VLDC significantlydecreased $(P<0.001)$ and simultensely there is increase in level of HDLs.Supercritical Fluid extraction of leaves of Aeglemarmelos(Am)possess significantantihyperlipidemic activity as compared to control group.
\end{abstract}

Keywords:Aeglemarmelos, Triton, Lipid profile, supercritical fluid extraction, Column chromatography

\section{Introduction}

Hyperlipidaemiais the greatest risk factor contributing to atherosclerosis[1-5] and occurrence of coronary heart disease and cerebrovascular accidents. Hence hypolipidemic[6] molecules are extensively usedas prophylactic agents to prevent such atherosclerosis induced disorders. But these hypolipidemic drugs [7]are not free from adverse effects. Pancreatitis due to moiety of statin is a well-known adverse effect and HMG-CoA reductase inhibitors have also been reported to cause hyperglycemia and FDA has given a warning to this effect. Many plant derivatives and domestic remedies have been screened for their hypolipidemic action. More than 70 medicinal plants have been documented to have significant hypolipidemic action. Beginning of last century evidence of lipid lowering[8] properties of medicinal plants has accumulated[9-11]. Ethno botanical information[12] indicates that more than 800 plants are used as traditional and remedies for the treatment of diabetes and lowering the lipid profile.Taking these finding forward is mandatory to develop new drugs in this area. Hence further research into identifying the active principle, conducting preclinical studies and if possible clinical studies is needed to evaluate the Antihyperlipidemic[13-16]activities of this plant.

\section{Materials And Methods}

Plant

Pharmacological studies have acknowledged the value of medicinal plants as potential source of bioactive compounds. Plants are known to be the source of many chemical compounds. Aeglemarmelos[17-29], commonly known as Bael, is a spiny tree belonging to the family Rutaceae Sub family:AurantioideaeGenus:Aegle.The leaves, roots, bark, seeds and fruits are edible and have medicinal values. The plant was authenticated by taxonomist and specimen (No.24/Am) is preserved in the department of Research Pharmacology, Bapuji Pharmacy College, Davangere (India).

\subsection{Supercritical extraction ${ }^{30}$}

The leaves of Aeglemarmelosas raw material were collected from a home garden. The leaves collected were washed with tap water to remove the dust and dirt. They were dried under sun drying for 3 hours. The dried leaves were powdered using a mixer to obtain powder of the raw leaves of Aeglemarmelos. Carbon dioxide (purity 99.99\%) as solvent contained in a dip tube cylinder is installed. Supercritical $\mathrm{CO}_{2}$ was obtained by using SFT-10 Supercritical Fluid Pump. The SFT-10 is a high precision carbon dioxide pump designed to deliver liquid carbon dioxide at pressures up to 10,000 psi (68.9 MPa). 


\subsection{Experimental procedure}

1. Turn on the power to the SFT-110 SFE unit.

2. Turn on the Peltier cooler at least thirty minutes prior to the start of the experiment.

3. Powder $\mathbf{5 0}$ grams of raw material i.e., dried Aeglemarmelos leaves.

4. Place the powdered Aeglemarmelos leaves into the SFT-110 Unit's processing vessel and seal.

5. Ensure that both the static/dynamic valve and restrictor valve are closed.

6. Open the carbon dioxide tank valve to allow the carbon dioxide to come into the unit ( $750 \mathrm{psi})$.

7. Set the oven temperature to $40^{\circ} \mathrm{C}$ and the restrictor block to $40^{\circ} \mathrm{C}$.

8. Set the pressure on the pump to $2000 \mathrm{psi}$. The pump should begin to actuate to pressurize the sample vessel. This will take between 12-15 minutes.

9. Once the pressure is up to $2000 \mathrm{psi}$, allow the sample to "soak" at that pressure for 15 minutes for $40^{\circ} \mathrm{C}$.

10. Open the static/dynamic valve to allow free flow of carbon dioxide through the restrictor valve. Adjust the restrictor valve to achieve about $24 \mathrm{ml} / \mathrm{min}$ of liquid carbon dioxide. Flow dynamically for 15 minutes. The pump should actuate and continue to maintain sample vessel pressure.

11. Close the static/dynamic valve and allow to "soaking" for an additional 15 minutes before repeating the dynamic flow step above. Repeat the static soak and dynamic flow step 5 more times.

12. Set the vessel temperature to ambient and set the pressure control down to ambient. Allow the unit to vent. When the vessel has reached ambient pressure, disconnect the inlet and outlet fittings, open the vessel and remove the Aeglemarmelos powder.

13. Similar procedure is carried out by varying the parameters such as temperature $\left(45^{\circ} \mathrm{C}, 50^{\circ} \mathrm{C}\right.$ and $\left.55^{\circ} \mathrm{C}\right)$, pressure (2000 psi, $3000 \mathrm{psi}$ and $4000 \mathrm{psi}$ ) and time (20, 25 and 40 minutes).

14. Phytochemical investigations of all extracts were carried out in order to detect the presence of the following class of compounds.

\subsection{Column chromatography}

The obtainedAeglemarmelos powder (10 gm) was subjected to column chromatographic methods. Thesupercritical fluid extract $(10 \mathrm{~g})$ was chromatographed over silica gel $(100-200 \mathrm{mesh})$ on column $55 \mathrm{~cm}$ length and $6 \mathrm{~cm}$ diameter. Elution was carried out with solvent mixtures of increasing polarities. Fractions were collected in $100 \mathrm{ml}$ portions and monitored by TLC (silica Gel adsorbent) and the fractions showing similar spots are pooled together. Elution with ethyl acetate: ethanol (EA: ET-OH (40: 60) gave brown crystalline solid $(450 \mathrm{mg})$ and named as AmF1. Similarly, elution with EA: ET-OH (30:70) yielded shiny brown coloured crystalline solid (1.5g) and was named as AmF2.

\subsection{Acute toxicology test ${ }^{31}$}

Acute toxicity study was performed using albino mice and doses were fixed as per OECD guideline No.423 and adopted CPCSEA protocol. Wister rats were made hyperilipidemic by injecting Triton-induced with a dose of $400 \mathrm{mg} / \mathrm{kg}$ (i.p), body weight is chilled citrate buffer ( $\mathrm{pH} 4.5$ ),

\subsection{Anti-hyperilipidemic activity}

Anti-hyperilipidemic activity of fractions $\mathrm{AmF} 1$ and $\mathrm{AmF} 2$ was performed on Triton-induced hyperlipidemic rats and was evaluated as per the method described by Moss11, Vogel12 and Hirch. Animals were divided in to six groups.

Group I: Served as solvent control.

Group II: Served as hyperlipidemic control (Triton-induced) $400 \mathrm{mg} / \mathrm{kg} .$, i.p.)

Group III: Received Statins $(5 \mathrm{mg} / \mathrm{Kg}, \mathrm{p} . \mathrm{o})$

Group IV: Received extract $(150 \mathrm{mg} / \mathrm{kg}, \mathrm{p} . \mathrm{o})$

Group V: Received extract $(75 \mathrm{mg} / \mathrm{Kg}$, p.o)

Group VI: Received fraction-1 (AmF1-75 mg/kg, p.o).

After 18 hours blood samples were collected from retro orbital sinus puncture under mild anaesthesia and transferred directly into centrifuge tube and allowed to clot at room temperature for 20-25 min and centrifuged at $2000 \mathrm{rpm}$. The supernatant clear serum thus obtained was transferred carefully with the help of micropipette into small test tubes for estimation. The serum concentration of total cholesterol, triglycerides, HDL-cholesterol and LDL-cholesterol were measured by standard procedure using auto analyser (RA 50 model, Miles India Limited).

\subsection{Statistical Analysis:}

Data were statically analyzed as meant \pm SEM and expressed as significant $\mathrm{P}<0.001$ using one way ANOVA 
followed by Dunnett's test.

\section{Results And Discussion}

The present investigation was focused on to evaluate antihyper-lipidemic activities of Aeglemarmelos leaves extract and its fraction AmF1.The result of the present study demonstrates the efficiency of Supercritical extract $\mathrm{Am}$ and fraction $\mathrm{AmF}$. The plant secondary metabolite like flavonoids are responsible to monitoring the normal lipid profile is Triton induced diabetic rats due to LA of lecithin acetyl transferase (LCAT), which regulates blood lipids and glucose concentration. The LCAT plays a key role in the incorporation of free cholesterol into HDL (this may increase HDL) and transferring it back to VLDL and LDL which are taken back later in liver cells.

Several investigation reports have positive witnessed that, increase in HDL-C is associated with decrease in coronary artery diseases. The result of the present study suggested that extract and fraction of $(\mathrm{AmF} 1)$ illustrate significant - antihypolipidemic activity $(\mathrm{P}<0.001 \mathrm{HS})$ and results were tabulated in Table -1 .

\subsubsection{Figure (1)}

\section{Figures And Tables}

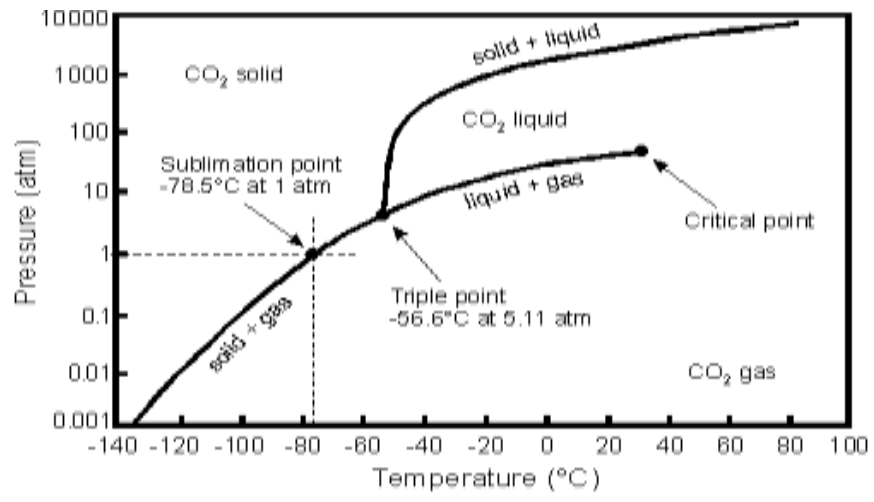

Pressure-T emperature phase diagram for $\mathrm{CO}_{\text {}}$

Fig. 1 Phase diagram for $\mathrm{CO}_{2}$

\subsubsection{Figure (2)Supercritical extraction set up.}

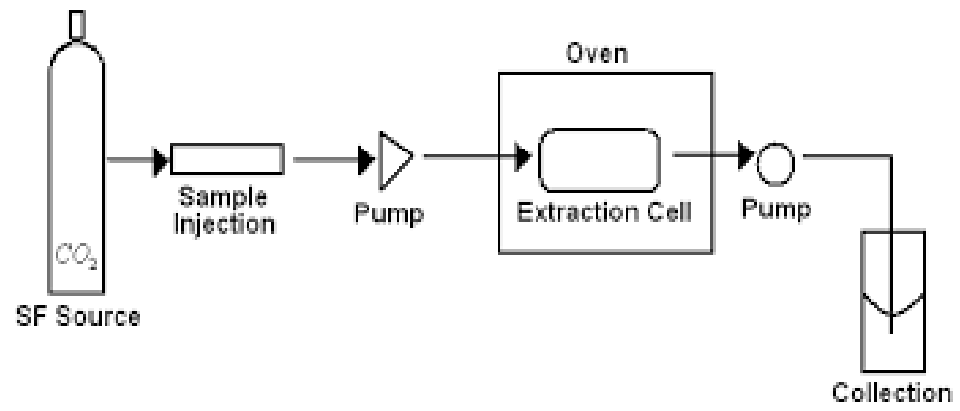

Fig. 2 Schematic diagram of Supercritical extraction set up.

\subsection{Tables}

Table 1 Estimation of serum parameters in Triton induced Hyperlipidemic rats.

\begin{tabular}{|l|l|l|l|l|l|}
\hline Group & TC & TG & HDL & LDL & VLDL \\
\hline Group I & $100.79 \pm 24.53$ & $90.25 \pm 35.95$ & $35.83 \pm 3.09$ & $65.00 \pm 38.54$ & $30.50 \pm 5.49$ \\
\hline Group II & $105.33 \pm 33.09$ & $94.29 \pm 39.79$ & $35.75 \pm 3.99$ & $70.21 \pm 39.02$ & $33.29 \pm 6.33$ \\
\hline Group III & $104.34 \pm 30.69$ & $92.70 \pm 40.36$ & $34.65 \pm 4.57$ & $67.17 \pm 37.59$ & $32.13 \pm 5.10$ \\
\hline Group IV & $103.17 \pm 29.39^{*}$ & $91.70 \pm 37.01^{*}$ & $34.04 \pm 3.67 *$ & $65.78 \pm 36.00^{*}$ & $32.35 \pm 4.22^{*}$ \\
\hline Group V & $104.35 \pm 31.28^{*}$ & $91.52 \pm 38.07^{*}$ & $36.30 \pm 3.92$ & $65.83 \pm 37.03^{*}$ & $31.30 \pm 4.52^{*}$ \\
\hline Group VI & $106.22 \pm 29.27^{*}$ & $91.30 \pm 38.37 *$ & $34.52 \pm 3.55$ & $66.48 \pm 37.57^{*}$ & $31.39 \pm 4.73^{*}$ \\
\hline
\end{tabular}

One way ANOVA followed by Dunnett's test *Indicates difference from groups $=p \leq 0.001$ HS.

\section{Conclusion}

The present investigation was focused on to evaluateantihyper-lipidemic activities of leaves extract 
ofAeglemarmelos and its fractions. The result of the present study demonstrates the efficiency and fractions of the Supercritical Fluid extracts of Aeglemarmelos $(A m)$ in lowering the serum cholesterol, triglycerides in rats. Secondary plant metabolites such as flavonoids, saponins, terpinoids, and alkaloids may be responsible for the antihyperlipidemic activity.

\section{Reference}

[1]. Gupta R et al. Younger age of escalation of cardiovascular risk factors in Asian Indian subjects. BMC Cardiovascular Disorders, 28(9),2009.

[2]. Kritchevsky D. Dietary protein, Cholesterol and atherosclerosis: A review of early history,J.Nutr 1995; 125 (Suppl 3): 5589-93.

[3]. Kjeldsen K, Stender S (1989) Calcium antagonists and experimental atherosclerosis. ProcSocExpBiol Med 190:219-228.

[4]. Knorr AM, Kazda S (1990) Influence of nifedipine on experimental arteriosclerosis. Cardiovasc Drugs Ther 4:1027-1032.

[5]. McCarthy PA (1993) New approaches to atherosclerosis: An overview. Med Res 13: 139-159.

[6]. VenuGopalaRaoKonda,Madhavi.E, Ruckmani.A And Venkataramana.Y. A Review on Medicinal Plants with Potential Hypolipidemic Activity. Int J Pharm Bio Sci; 4(4) 2013 Oct : (P) 729 - 740

[7]. Holmes WL (1964) Drugs affecting lipid synthesis. In: Pao-letti R (ed) Lipid Pharmacology, Academic Press, New York, London, chapter 3, pp 131-184.

[8]. Illingworth DR (1987) Lipid-lowering drugs. An overview of indications and optimum therapeutic use. Drugs 33:259-279.

[9]. Belfiore F, Mogensen CE (eds): New Concepts in Diabetes and Its Treatment. Basel, Karger, 2000, pp 1-2.

[10]. M. Silinki, J. Tuomilehtoii, J.C. Mbanyaiii, K.M. VenkatNarayaniv, Judy Fradkinv and G. Roglicvi. Research priorities; Prevention and control of diabetes with a focus on low and middle income countries. Working paper series. WHO Meetings on Development of a prioritized Research Agenda for Prevention and Control of Noncommunicable Diseases 2008, 2009, 2010, World Health Organization, Geneva Switzerland.R.K. Goyal. Traditional systems of Medicine. cpp 280.

[11]. Definition and diagnosis of diabetes mellitus and intermediate hyperglycaemia. Geneva, World Health Organization, 2006.

[12]. Van Heek M, Farley C, Copton DS, Hoos LM, Smith-Torhan A, Davois HR Ezetimibe potently inhibits cholesteril absorption but does not affect acute hepatic or intestinal cholesterol synthesis in rats.Br J Pharmacol2003,138:1459-1464.

[13]. Moss JN and Dajani EZ, Antihypoperlipidemic agents in screening methods in Pharmacology, edited by R.A.Turner, P.A.Hebban, Academic Press, New York: 197:

[14]. Devi R and Sharma DK, Hypolipidemic effect of different extracts of Clerodendrumcolebrookinumwalp in normal and high fat diet fed rats : J.Ethnopharmacol (2004) : 90:63.

[15]. Van Heek M, Farley C, Compton DS, Hoos L, Alton KB, Sybertz EJ, Davis HR Comparison of the activity and disposition of the novel cholesterol absorption inhibitor, SCH5835, and its glucuronide, SCH60663. Br J Pharmacol2000, 129:1748-1754.

[16]. S Brijesh, Poonam Daswani, Pundarikakshudu Tetali, Noshir Antia,Tannaz Birdi;Studies on the antidiarrhoeal activity of Aeglemarmelos unripe fruit: Validating its traditional usage; The official of the International society for complementary medicine Research (ISCMR);47(9);2009.

[17]. Sharma PK, Bhatia V, Bansal N, Sharma A.A review on bail tree. Natural Product Radiance; 6(2) 2007: 171-178.

[18]. S Balakumar, S Rajan, T Thirunalasundari, and S Jeeva4; Antifungal activity of Aeglemarmelos (L.) Correa (Rutaceae) leaf extract on dermatophytes;Asian pacific Journal of Tropical Biomedicine; 1(4); 2011; p-309-312.

[19]. MalviyaRishabha, Kumar Ajay, Singh Anupama, KulkarniGT;Pharmacological Screening, Ayurvedic values and Commercial Utility of Aeglemarmelos; International Journal of Drug development\& Research; 4(1); 2012; p-28-37.

[20]. Phatru Patel, Syed Mohammed BasheeruddinAsdaq; Immunomodulatory activity of methanolic fruit extract of Aeglemarmelos in experimental animals; Saudi Pharmaceutical Journal; 18(3); 2010; p-161-165.

[21]. Sahare KN, Anandhraman V, MeshramVG,Meshram SU, Reddy MV, Tumane PM, et al. "Anti-microfilaria activity of methanolic extract of Vitexnegundo and Aeglemarmelos and their phytochemical analysis."Indian Journal of Experimental Biology. 2008; 46(9022):128-131.

[22]. Yaheya M, Ismail M. Clinical evaluation of antidiabetic activity of Trigonella seeds and Aeglemarmelos leaves. World Applied Sciences Journal; 7 (10): 2009, 1231-1234.

[23]. Chauhan A, Agarwal M, Kushwaha S, Mutreja A. Antifertility studies of Aeglemarmelos Corr., an Indian medicinal plant on male albino rats. Egyptian Journal of Biology, 2008; 10: 28-35.

[24]. Singanan V, Singanan M, Begum H. The hepatoprotective effect of bael leaves (Aeglemarmelos) in alcohol induced liver injury in albino rats. International Journal of Science \& Technology, 2(2), 2007: 83-92.

[25]. www.hillgreen.com/pdf/AEGLE\%20MARMELOS.pdf

[26]. Aeglemarmelos (Bael Fruit) Herbs - Information and Medicinal Benefits. Online vitamins guide. www.online-vitamins-guide.com

[27]. Siddique NA, Mujeeb M, Najmi AK, Akram M. Evaluation of antioxidant activity, quantitative estimation of phenols and flavonoids in different parts of Aeglemarmelos. African Journal of Plant Science; 4 (1), 2010: 001-005.

[28]. Dinesh Kumar Sekar, Gaurav Kumar, L. Karthik and K. V. BhaskaraRao;A review on pharmacological and phytochemical properties of Aeglemarmelos (L.) Corr. Serr. (Rutaceae);Asian Journal of Plant Science and Research, 1 (2): 2011, 8-17

[29]. Ghosh MN. Fundamentals of experimental Pharmacology, Toxicity studies. Scientific Book agency, Calcutta, (1984); 2; 44-52.

[30]. RabinarayanParhi, Padilama Suresh; super critical fluid extraction: a review; advanced pharmaceutical science and technology; 1(1) p-13 